\title{
Insectos descortezadores (Coleoptera: Curculionidae) Y CAMBIO CLIMÁTICO: PROBLEMÁTICA ACTUAL Y PERSPECTIVAS EN LOS BOSQUES TEMPLADOS
}

\author{
Ek del-Val ${ }^{1,2 *}$ y Cuauhtémoc Sáenz-Romero ${ }^{3}$ \\ ${ }^{1}$ Escuela Nacional de Estudios Superiores Unidad Morelia, ${ }^{2}$ Instituto de Investigaciones en Ecosistemas y \\ Sustentabilidad, Universidad Nacional Autónoma de México, Antigua Carretera a Pátzcuaro \# 8701, Col. Ex- \\ Hacienda de San José de La Huerta, C.P. 58190, Morelia, Michoacán, México, ${ }^{3}$ Instituto de Investigaciones \\ Agropecuarias y Forestales, Universidad Michoacana de San Nicolás Hidalgo, Av. San Juanito Itzícuaro s/n, \\ Col. Nueva Esperanza, C.P. 58330, Morelia, Michoacán, México. E-mail: *ekdelval@cieco.unam.mx
}

\begin{abstract}
Resumen
En los bosques templados están sucediendo modificaciones importantes debido al cambio climático; en latitudes polares va en aumento su área de distribución, mientras que en las tropicales se reduce por incremento en la temperatura y las sequías. Uno de los agentes bióticos reguladores de los bosques templados son los insectos descortezadores que ocasionan la mortalidad de ciertos árboles. Estos insectos han aumentado en número, favorecidos por el cambio climático y las consecuencias sobre los bosques no se han dejado esperar. En épocas recientes en el hemisferio norte la mortalidad masiva de coníferas debida a la sinergia negativa entre cambio climático e insectos descortezadores ha sido evidente. En México, también se han experimentado infestaciones por descortezadores nunca antes vistas; por lo que se está tratando de entender las interacciones involucradas entre el cambio climático, la salud forestal y los insectos descortezadores, para detectar las áreas con mayor susceptibilidad al ataque por estos insectos y proponer medidas de manejo que disminuyan las afectaciones. Palabras Clave: bosques de coníferas, cambio climático, sequía, Scolytinae.
\end{abstract}

\section{Bark-beetles (Coleoptera: Curculionidae) and climate change: current situation and perspectives for temperate forests}

\begin{abstract}
Temperate forests are experiencing important modifications due to climate change, in polar latitudes they are increasing their distributional range while in tropical latitudes they are decreasing, due to higher temperature and droughts. Bark beetles are considered one of the biotic agents that regulate temperate forests because they kill some trees. These insects have increased in abundance, favored by climate change, and the consequences for temperate forests are evident. In recent times, the northern hemisphere has suffered a massive coniferous mortality due to the negative synergy between climate change and bark beetle abundance. Mexico has also experienced bark beetle infestations never seen before; this is why it is important to understand the interactions between climate change, forest health and bark beetle abundance to visualize the most important bark beetle susceptible areas in Mexico. Also with this information we could propose management strategies to diminish bark beetle impacts.
\end{abstract}

Key Words: coniferous forests, climate change, drought, Scolytinae.

Nota: Artículo recibido el 29 de noviembre de 2016 y aceptado el $\mathbf{2 7}$ de abril de 2017. 


\section{INTRODUCCIÓN}

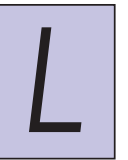

os bosques templados en el mundo, constituyen un ecosistema que abarca el $15 \%$ de la superficie terrestre, siendo importante por la provisión de servicios ambientales para los humanos. En México, los bosques templados están dominados por coníferas del género Pinus con la mayor diversidad de especies de este género a nivel mundial, convirtiéndose el país en el centro de origen y diversificación de este grupo (Challenger \& Soberón, 2008). El género Pinus se originó en el Cretácico inferior (hace $\sim 145$ millones de años) y en la actualidad cuenta con 111 especies, de las cuales 46 especies con 3 subespecies y 22 variedades están presentes en México y de éstas 55\% son endémicas (Sánchez-González, 2008). Los bosques de pino en México, se encuentran distribuidos principalmente en las sierras con altitudes entre los 1,500 a los 4,000 msnm. Dependiendo de la altitud, la exposición y la latitud en la que se encuentren, se congregan en diferentes comunidades, formando bosques de coníferas, bosques de pino-encino o bosques de encino-pino, según la dominancia de cada género (Rzedowski, 1978).

Además de su importancia filogenética y biogeográfica, el género Pinus tiene una gran relevancia social, ya que por su utilidad se ha convertido en una parte significativa del desarrollo de México y hoy en día muchas comunidades rurales dependen de los bosques como forma de vida.

El manejo de los bosques de pino en México, se relaciona principalmente con la explotación maderera. Según los datos de la Comisión Nacional Forestal (CONAFOR) en 2014 se cosecharon 4.3 millones de $\mathrm{m}^{3}$ de pino y representaron un ingreso de 6,039 millones de pesos (SEMARNAT, 2015); además, los bosques de pinos proveen otro tipo de recursos no maderables como la resina, animales para alimento, plantas medicinales y ornamentales y de manera muy importante de combustible para un número importante de hogares. Se ha calculado que 27 millones de personas dependen de la leña para cocinar tanto en el sector urbano como el rural (Berrueta Soriano, 2007). Los bosques de pino en México, son fundamentales también para la provisión de servicios ambientales como son: la captura de agua, captura y almacén de carbono, regulación de la temperatura, además de ser un resguardo de la biodiversidad y belleza escénica (CONABIO, 2009). Sin embargo, a pesar de que los bosques templados mexicanos están sujetos a un uso importante y una porción creciente de ellos se encuentra bajo algún programa de manejo, existe una gran disparidad en cuanto a su éxito. Si bien hay comunidades con una organización y manejo ejemplar, como Nuevo San Juan Parangaricutiro en Michoacán e Ixtlán de Juárez en Oaxaca, en numerosas comunidades forestales existe la tala ilegal e incendios no controlados, aún con planes de manejo vigentes, lo cual pone en riesgo el desarrollo sustentable de las regiones de bosque templado en el país (Bray et al., 2007).
Actualmente, los bosques de pino en México, cubren aproximadamente 10 millones de Ha, no obstante, de acuerdo con Challenger \& Soberón (2008), solamente el $50 \%$ se encuentra en buen estado de conservación y el resto presenta algún tipo de deterioro. Debido a la sobrexplotación y cambio de uso de suelo, algunas especies están dañadas y requieren de protección legal específica para su conservación. De hecho, se ha registrado que al menos 20 taxa de pinos están en alguna categoría de riesgo y dos de ellos: Pinus maximartinezii y P. muricata se encuentran en peligro de extinción (Sánchez-González, 2008).

\section{DistuRBio en lOS BOSQUES TEMPLADOS}

Los bosques templados presentan regímenes de disturbios variables. En algunas regiones el fuego es el principal agente que permite la regeneración de especies no dominantes y elimina a los árboles débiles e incluso algunas especies de pino dependen de las altas temperaturas generadas por el fuego para que se abran los conos donde se alojan sus semillas (Pausas \& Keeley, 2009). Debido a que la gran mayoría de los bosques de pino son manejados por el hombre, la frecuencia e intensidad de los fuegos naturales se han modificado. En algunos lugares se suprimen los fuegos, para impedir la pérdida del arbolado, mientras que en otros, se han promovido en exceso los incendios, para realizar cambio de uso de suelo con fines agrícolas o ganaderos (Rodríguez-Trejo, 2008; Pérez-Salicrup et al., 2016).

\section{DesCORTEZADORES}

Los escarabajos descortezadores son coleópteros herbívoros que pertenecen a la familia Curculionidae de tamaño muy pequeño, su cuerpo oscila entre los 0.1 y $0.6 \mathrm{~cm}$ y presentan el hábito de ser endófitos, cavan galerías por debajo de la corteza de los árboles para alimentarse. Hay dos grandes grupos: los pertenecientes a la subfamilia Scolytinae que se alimentan directamente del floema de los árboles y los escarabajos conocidos como Ambrosiales, dentro de la subfamilia Paltipodinae, que además de perforar la corteza, cultivan hongos ambrosiales y no se alimentan directamente del árbol hospedero, sino de los hongos que cultivan en su interior (Farrel et al., 2001). Entonces, es importante diferenciar que aunque los dos grupos de Curculionidae viven dentro de los árboles, solamente unos se alimentan directamente del floema (Scolytinae), mientras que los otros de los hongos que cultivan (Platipodinae). En ambos casos, los escarabajos adultos cavan un túnel en la corteza de los árboles para ovipositar por debajo de ella. En algunas especies de ambos grupos solamente la hembra cava y en otras la ayuda el macho. Una vez dentro del árbol y después de aparearse, las hembras hacen un túnel vertical en donde van ovipositando, separando los huevos con cierta distancia entre sí, para que no haya interferencia entre las larvas al momento de alimentarse. Cuando emergen las larvas conforme van alimentándose, cavan túneles longitudinales alejándose del túnel parental y comen hasta que adquieren la talla adecuada para llevar a cabo la metamorfosis, salir del árbol como adultos y completar su ciclo de vida. Cada especie 
de descortezador deja un patrón de galerías diferente en la madera del árbol (Wood, 1982). En el caso de los escarabajos ambrosiales, cada especie tiene una asociación mutualista con un hongo en particular, por lo que cuando los jóvenes adultos emergen de los árboles para buscar una pareja y aparearse, llevan consigo esporas de su hongo simbionte con el fin de compartir con la nueva generación el hongo que les proporcionará alimento, asegurando así el bienestar de la nueva generación (Wood, 1982). Por lo general, la mayoría de las especies de escarabajos descortezadores atacan árboles debilitados por la edad, la sequía, el fuego, enfermedades o daño mecánico, sin embargo, hay especies agresivas como Dendroctonus frontalis (Fig. 1)y Dendroctonus ponderosae que, cuando sus poblaciones son muy grandes, pueden infestar árboles sanos al realizar ataques masivos sobre ellos en superficies extensas (Billings et al., 2004; Bentz et al., 2010).

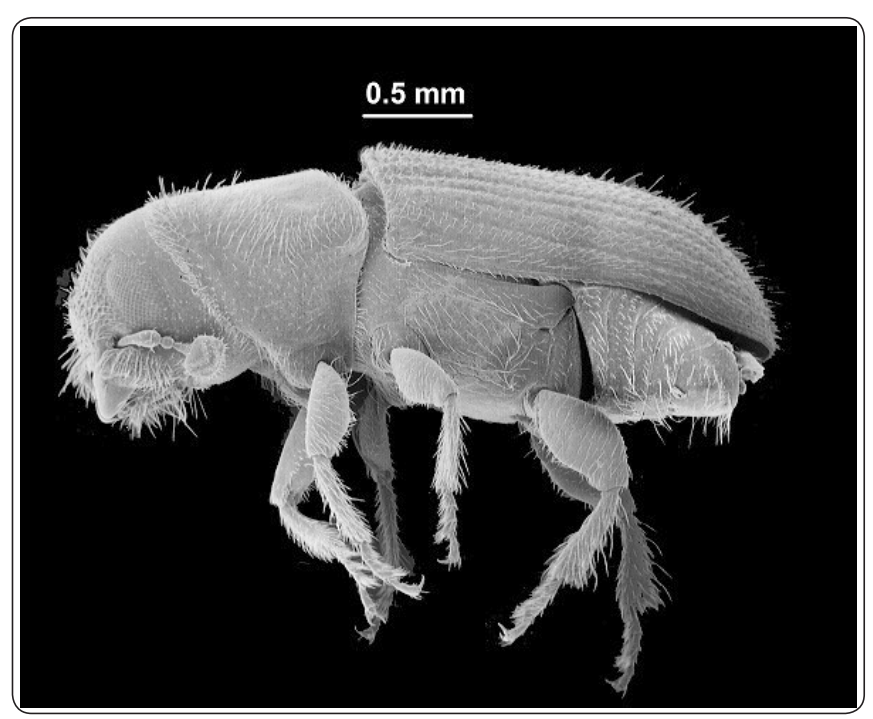

Figura 1. Escarabajo descortezador del pino, Dendroctonus frontalis. Fotografía cortesía del Dr. Thomas Atkinson.

En el mundo hay descritas alrededor de 3,000 especies de escarabajos descortezadores y en México se encuentran 870 de éstas distribuidas en 87 géneros, aunque es probable que esta cifra aumente, porque aún se siguen describiendo nuevas especies (Atkinson, 2013).

Los descortezadores tienen una función primordial en la dinámica de los bosques templados, al promover el recambio de especies vegetales permitiendo el establecimiento de otras que son menos competitivas cuando eliminan a algunos individuos dominantes. Sin embargo, son pocas las especies de insectos que pueden causar la mortalidad de los árboles por si mismas, la mayoría no matan al árbol hospedero y se mantienen en poblaciones pequeñas como agentes de saneamiento natural del bosque (Christiansen \& Bakke, 1988). En México, el número de especies de escarabajos descortezadores que causan mortalidad de las coníferas, se reduce a menos de 20, la mayoría pertenecen al género Dendroctonus y algunas especies al género Ips (Cibrián-Tovar et al., 1995; Salinas-Moreno et al., 2004; Fonseca et al., 2008).

\section{Cambio climático}

Los cambios ambientales que el planeta Tierra está experimentando debido a las actividades antropogénicas, han tenido diferentes repercusiones sobre los ecosistemas. En promedio la temperatura global del planeta se ha incrementado entre 0.5 $1^{\circ} \mathrm{C}$ y con ello los fenómenos meteorológicos extremos como tormentas o huracanes (World Metereological Organization, 2013). En México, también se ha observado un aumento generalizado en la temperatura en épocas recientes (SáenzRomero et al., 2010) independientemente de las oscilaciones relacionadas con el fenómeno meteorológico de El Niño (Pavia et al., 2009). Las predicciones para el año 2030, indican que el país experimentará un creciente nivel de temperatura de $1.4^{\circ} \mathrm{C}$ en promedio y una disminución en la precipitación promedio de 5.6\% (Sáenz-Romero et al., 2010). En algunas regiones la temperatura ha sido incluso mayor y se ha combinado con una reducción en la precipitación, propiciando condiciones de sequía inusuales. En particular los efectos directos del cambio climático sobre la vegetación son evidentes en todo el mundo (Walther et al., 2002; Parmesan \& Yohe, 2003). Por ejemplo, las zonas áridas y semiáridas están expandiéndose (Archer et al., 1995), el límite latitudinal de los bosques templados se ha recorrido hacia los polos (Grace et al., 2002) y varios tipos de vegetación, así como varias especies en particular, han migrado altitudinalmente en tiempos recientes (Lenoir et al., 2008). Las poblaciones de animales también han experimentado cambios importantes; por ejemplo, en Gran Bretaña se ha reportado que 22 especies de mariposas han expandido su ámbito hogareño hacia latitudes más frías (Parmesan et al., 1999), mientras que en España, 16 especies de mariposas cambiaron su distribución altitudinal (subieron casi $200 \mathrm{~m}$ ) lo que representa un aumento considerable de su hábitat óptimo (Wilson et al., 2005).

\section{Cambio Climático y descortezadores}

En particular, el cambio climático global en los bosques templados ha propiciado una disminución en la intensidad de los inviernos y se ha reducido el número de días con temperaturas bajo cero, por lo que se consideran como uno de los ecosistemas más vulnerables ante el fenómeno (González et al., 2010). Varios estudios han documentado que existe un desacoplamiento entre las condiciones climáticas actuales y los requerimientos ambientales de los bosques templados (Smith et al., 1992; Brolsma, 2010; Reich et al., 2016), y que este desacoplamiento se acentuará hacia el futuro con las predicciones climáticas para las regiones templadas (Rehfeldt et al., 2012). En términos biológicos, esta situación de alteración climática, ha dado lugar a que el crecimiento de los árboles, pueda extenderse por un mayor periodo de tiempo en las regiones 
más frías (Bentz et al., 2010), mientras que los árboles en el límite de distribución altitudinal inferior y latitudinal hacia el Ecuador, se encuentran más estresados por sequía ocasionando mortalidades masivas por falta de agua (Allen et al., 2010; Mátyás, 2010; Worral et al., 2013; Allen et al., 2015). En estos mismos bosques, la regulación de las especies de herbívoros relacionada con la presencia de las bajas temperaturas, ha disminuido considerablemente dando como resultado que las poblaciones de algunos insectos herbívoros aumenten y se conviertan en plaga al expandir sus poblaciones, en particular Dendroctonus ponderosae y Dendroctonus frontalis (Bentz et al., 1991; Safranyik \& Linton, 1998; Raffa et al., 2008). Este es el caso de los escarabajos descortezadores que ha sido bien documentado en Estados Unidos, Canadá y Europa (Ungerer et al., 1999). Solamente en Canadá, en el año 2008 se reportó una infestación epidémica en los bosques de la Provincia de Columbia Británica, con un brote de Dendroctonus ponderosae que en menos de 10 años acumuló 14 millones de hectáreas afectadas donde hubo una mortalidad desproporcionada en cinco especies de pinos (Safranyik et al., 2010) En Colorado y Arizona, el grupo de investigación de Negrón et al., 2009, modelaron en el paisaje los factores determinantes que dieron lugar a una creciente mortalidad de Pinus ponderosa asociada a descortezadores (Dendroctonus ponderosae, Ips lecontei, I. pini, I. calligraphus, I. latidens, I. knausi e I. integer) y encontraron que los sitios con mayor sequía ubicados en altitudes menores presentaban mayores mortalidades arbóreas (Negrón \& Popp, 2004; Negrón et al., 2009). En Europa se han experimentado situaciones similares, particularmente entre 1980 y 1985 hubo sequías recurrentes en el centro del Continente que desencadenaron en una infestación importante de varios géneros como (Ips, Dendroctonus, Scolytus y Tomicus), sobre diversas coníferas (Picea spp., Pinus spp., Abies spp.), con una mortalidad inusual de árboles localizados en rangos altitudinales menores (Schutt \& Cowling, 1985).

La problemática, que los escarabajos descortezadores representan para los bosques templados, en las condiciones actuales de cambio climático, son un ciclo de retroalimentación negativa con impacto muy fuerte sobre los ecosistemas templados y que continúa en aumento conforme las temperaturas y la sequía se acentúan (Logan et al., 2003; Anderegg et al., 2015). Es decir que, al aumentar la temperatura, las poblaciones naturales de descortezadores incrementan el número de individuos y su capacidad de infestación, mientras que los árboles se ven estresados por la sequía y disminuyen su capacidad de defenderse del ataque de estos insectos (Fig. 2). Como se explicó anteriormente, los árboles en los límites xéricos de distribución de estos ecosistemas regularmente se encuentran sometidos a un estrés anormal y por lo tanto están fisiológicamente disminuidos, lo que implica que tienen una menor capacidad de defenderse del ataque de los insectos herbívoros y en

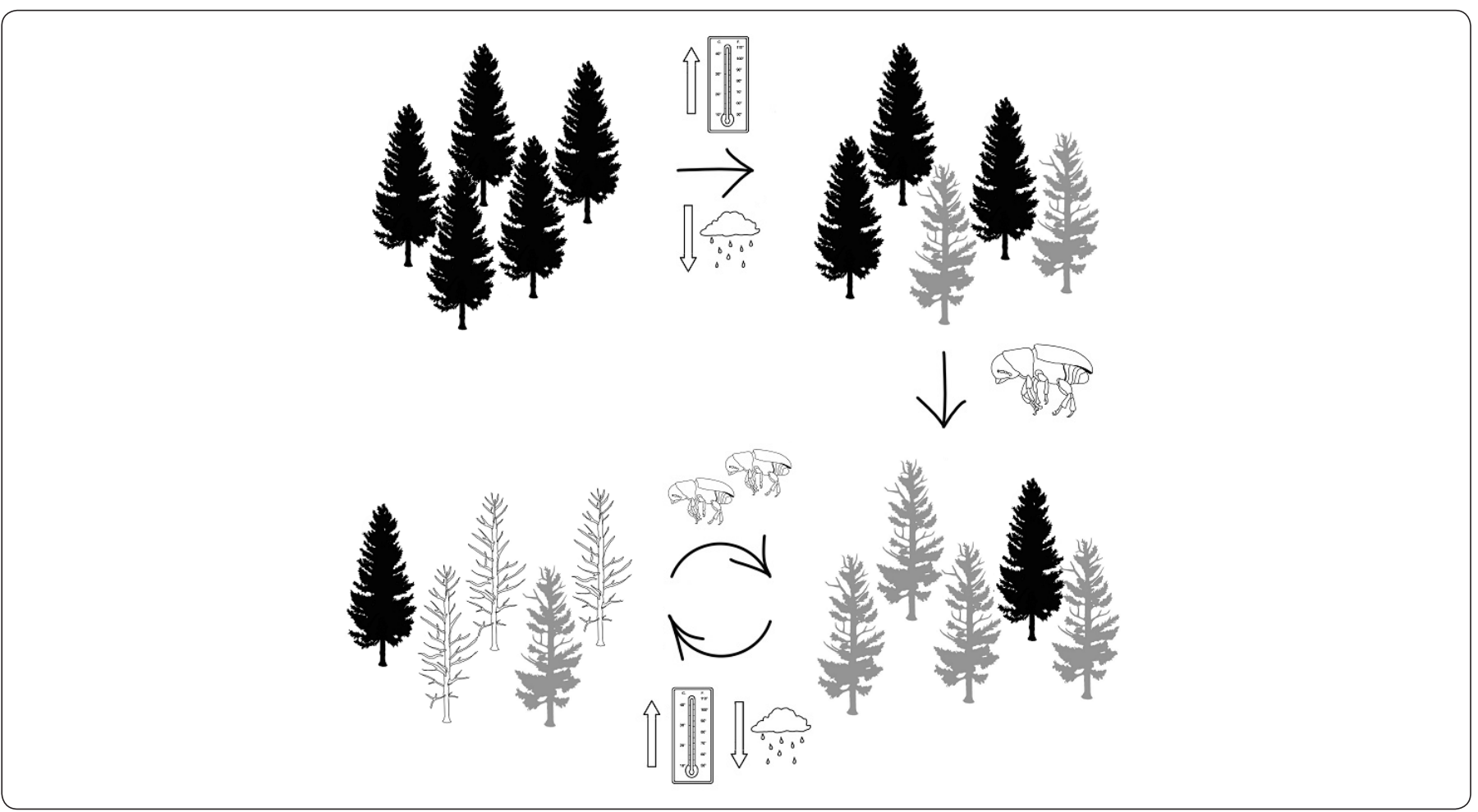

Figura 2. Ciclo de retroalimentación negativa entre los cambios climáticos y la incidencia de descortezadores sobre la salud del bosque. Realizada por Regina González. 
condiciones extremas se mueren. Los pinos se defienden de ellos mediante la producción de resinas, de manera que un árbol sano al ser perforado por un insecto, libera un torrente de resina que impide a éstos penetrar a sus tejidos (Fig. 3); sin embargo, cuando el árbol está débil presenta menos resina al reducirse ésta en los ductos por donde pasa en el xilema o incluso deja de producirla y los insectos penetran y acceden fácilmente al floema, terminando por matar al árbol (Gaylord et al., 2015). Por otro lado, el aumento en la temperatura ambiental además de disminuir la mortalidad de los insectos en el invierno (Safranyik \& Linton, 1998; Faccoli, 2002), acelera su ritmo metabólico, inhibe la diapausa y ha permitido que aumenten el número de generaciones que tienen por año (Bentz et al., 1991; Bentz et al., 2010). Algunos estudios, han reportado por ejemplo que en el sur de Italia, donde el clima es menos extremo que en el norte, Ips typographus presenta dos generaciones al año en lugar de una que era lo usual (Faccoli, 2002). Otros estudios han encontrado que las poblaciones de Pinus contorta del norte de la Columbia Británica que no habían estado expuestas a incrementos poblacionales de Dendroctonus ponderosae, son más susceptibles a los nuevos ataques que las poblaciones donde coincidían ambas especies, por lo que prevén que las explosiones poblacionales sigan teniendo efectos muy importantes en las poblaciones norteñas (Cudmore et al., 2010). En épocas recientes, estudios relacionados con los grandes brotes de Dendroctonus ponderosae en Canadá, estimaron la modificación en el sumidero de carbono que normalmente representan los bosques templados al producirse una mortalidad masiva de árboles. Kurz et al. (2003), calcularon que los bosques de Columbia Británica, en los años de fuerte infestación, no solamente dejaron de absorber el bióxido de carbono atmosférico, sino que se convirtieron en una importante fuente de producción de bióxido de carbono equivalente al $\sim 75 \%$ de los gases emitidos anualmente en todo Canadá, debido a los incendios forestales. Por otro lado, en California, Etaough Jones et al. (2004), encontraron que los pinos en sitios con alta contaminación por nitrógeno son más susceptibles al ataque por descortezadores. De manera que la retroalimentación negativa, con el aumento en las emisiones de gases de efecto invernadero, debido a la mortalidad masiva de árboles y calentamiento global, también tenderá a acrecentarse junto con la deposición de nitrógeno.

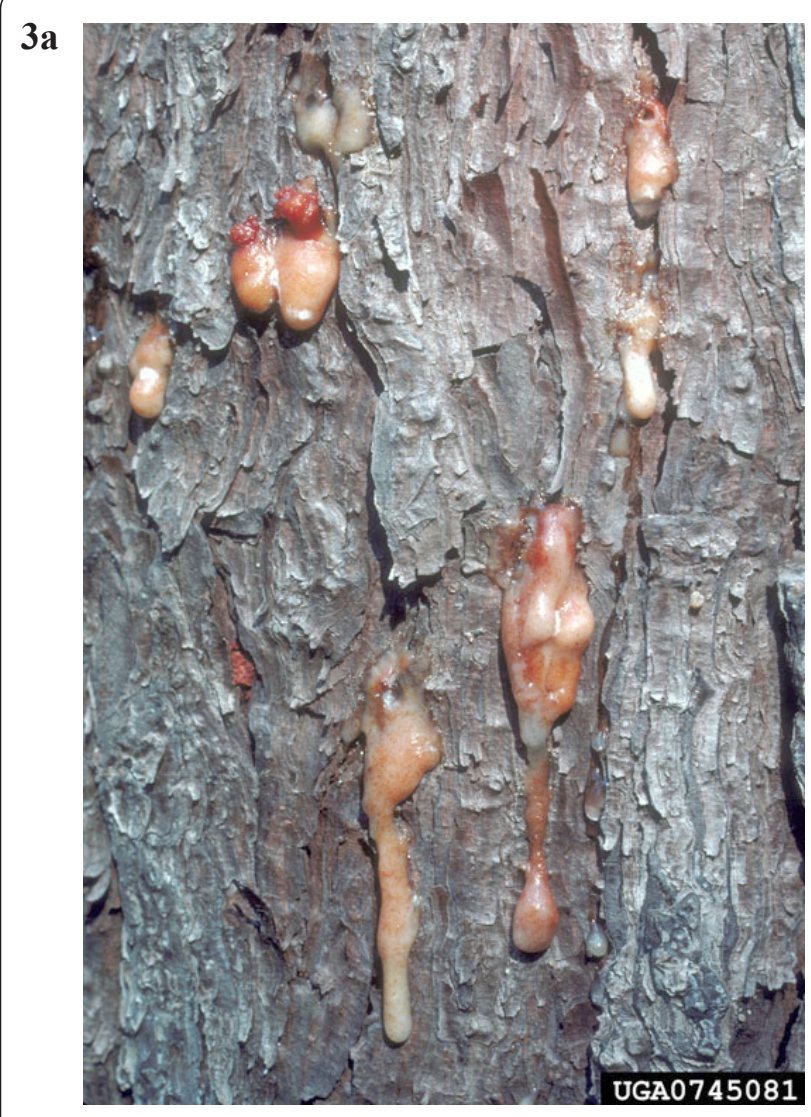

3b

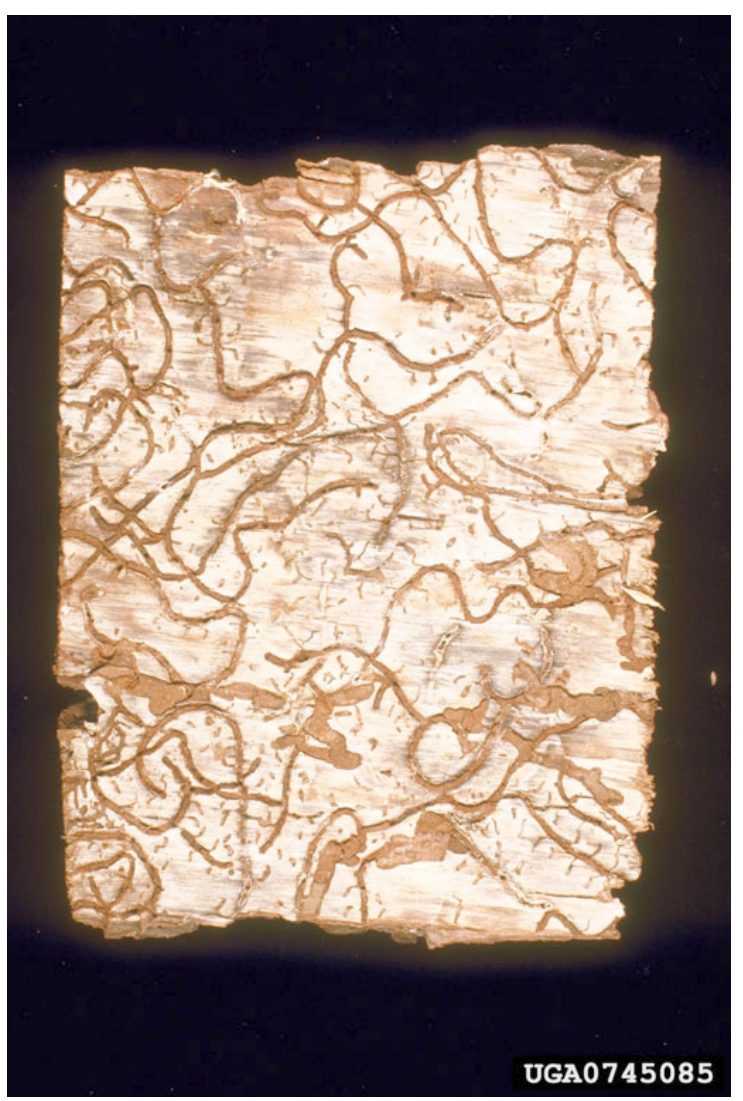

Figura 3. a) Tronco de pino atacado por descortezadores. Se muestran los grumos de resina producidos por el árbol ante la perforación de los escarabajos, fotografía cortesía de Erich G. Vallery, USDA Forest Service - SRS-4552, Bugwood.org. b) Galerías formadas por D. frontalis debajo de la corteza de los pinos, fotografía cortesía de W.H. Bennett, USDA Forest Service, Bugwood.org 


\section{Descortezadores en México ante el Cambio climático GLOBAL}

Por lo hasta aquí expuesto, los bosques templados de México, no son la excepción ante las consecuencias del cambio climático y su relación con las plagas forestales (Návar, 2015). En épocas recientes, se han documentado brotes de escarabajos descortezadores que afectan grandes extensiones de bosques. Por ejemplo, en el año 2013 el 12\% de los bosques de pinos presentaron brotes (Sistema Nacional de Información Ambiental), en particular en ese mismo año el $25 \%$ de los bosques de Durango y $18 \%$ de los bosques de Chihuahua tuvieron una afectación importante.

Históricamente, los mayores brotes de descortezadores en México, han ocurrido en el Eje Neovolcánico transversal, zona que coincide con la mayor diversidad de pinos, pero no con la mayor diversidad de especies de descortezadores del género Dendroctonus (Salinas-Moreno et al., 2010). En Michoacán, se ha reportado un aumento en la incidencia de brotes de estos insectos: en la Reserva de la Biosfera de la Mariposa Monarca en los años 2007-2008, cuando se presentó una sequía importante, se registraron infestaciones de Scolytus mundus causando una alta mortalidad sobre árboles de oyamel (Manzo-Delgado et al., 2013). En la comunidad forestal de Nuevo San Juan Parangaricutiro, también se identificó un incremento en el número de brotes de descortezadores, por lo que se realizó un estudio para evaluar tanto la incidencia de éstos en el bosque como el daño. En dicho trabajo se encontró la existencia de una relación significativa entre la abundancia de este tipo de insectos y las máximas temperaturas (Rubín-Aguirre et al., 2015); en la comunidad donde se realizó el estudio existe una mayor riqueza y abundancia de descortezadores secundarios en altitudes bajas (mediada por las máximas temperaturas) y también un mayor daño sobre el arbolado. Este patrón, aporta información para interpretar el aumento de descortezadores en altitudes menores de los bosques de pino, así como corroborar que los pinos están debilitados en los sitios con mayor temperatura y también que la abundancia de insectos es mayor a bajas altitudes, siendo el resultado un aumento en la probabilidad de focos de infestación, sobre todo si este patrón se corrobora con descortezadores más agresivos. Adicionalmente, es importante resaltar que la abundancia y distribución de los descortezadores también está muy relacionada con factores bióticos como la comunidad de depredadores en cada lugar (Reeve, 1997; Ryall \& Fahrig, 2005), así como con la composición de la vegetación de cada bosque en particular (Winter et al., 2015; Dhar et al., 2016). En México, hay muy pocos estudios que hayan realizado evaluaciones sobre estos factores, por lo que sin duda representan un área de oportunidad significativa.

\section{Conclusiones}

Las predicciones de cambio climático en México son drásticas para los bosques templados y son pocos los estudios que documenten la distribución espacio-temporal de la infestación, magnitud de los brotes de descortezadores en ellos y su posible asociación con el incremento de la temperatura y cambio en el patrón de lluvias. Debido a las evidentes consecuencias negativas que se han registrado en otros países como: la mortalidad masiva de árboles, el aumento en la emisión de gases de efecto invernadero, así como las repercusiones para las comunidades que viven de los bosques con la consecuente pérdida de su sustento de vida, es fundamental encaminar esfuerzos para entender mejor la dinámica de los descortezadores en el país y poder proponer no sólo estrategias de manejo que permitan prevenir y controlar su incidencia, sino también, para disminuir los costos por daños en la actualidad y en el futuro próximo.

\section{Agradecimientos}

Agradecemos las opiniones de dos revisores anónimos que fortalecieron el manuscrito. Este artículo forma parte del proyecto de investigación CONACYT-CONAFOR 2014CO1-234547.

\section{Referencias}

Allen, C. D., Breshears, D. D. \& McDowell, N. (2015). On underestimation of global vulnerability to tree mortality and forest die-off from hotter drought in the Anthropocene. Ecosphere 6:1-54. DOI: 10.1890/ES15-00203.1

Allen, C. D., Macalady,A. K., Chenchouni,H., Bachelet, D., McDowell, M., Vennetier, T., Kitzberger, A., Rigling, D. D., Breshears, E. H., Hogg, González, P., Fensham, R., Zhang, J., Castro, J., Deminova, N., Lim, J. H., Allard, G., Running, S. W., Semerci, A. \& Cobb, N. S. (2010). A global overview of drought and heat-induced tree mortality reveals emerging climate change risks for forests. Forest Ecology and Management 259:660-684. DOI: 10.1016/j. foreco.2009.09.001

Anderegg, W. R. L., Hicke, J. A., Fisher, R. A., Allen, C. D., Aukema, J., Bentz, B. J., Hood, S., Lichestein, J. W., Macalady, A. K., McDowell, N., Pan, Y., Raffa, F. K., Sala, A., Shaw, J. D., Stephenson, N. L., Tague, C. \& Zeppel, M. (2015). Tree mortality from drought, insects, and their interactions in a changing climate. New Phytologist 208:674-683. DOI: 10.1111/nph.13477

Archer, S., Schimel, D. S. \& Holland, E. A. (1995). Mechanisms of shrubland expansion: land use, climate or $\mathrm{CO}_{2}$ ? Climatic Change 29:91-99. DOI: 10.1007/BF01091640

Atkinson, T. H. (2013). Estado de conocimiento de la taxonomía de los escarabajos descortezadores y ambrosiales de México(Coleoptera: Curculionidae: Scolytinae). Pages 13-27 in XVI Simposio de Parasitología Forestal. Comisión Nacional Forestal.

Bentz, B. J., Logan, J. A. \& Amman, G. D. (1991). Temperaturedependent development of the mountain pine beettle (Coleoptera: Scolytidae) and simulation of its phenology. The Canadian Entomologist 123:1083-1094.

Bentz, B. J., Régnière, J., Fettig, C. J., Hansen, E. M., Hayes, J. L., Hicke, J. A., Kelsey, R. G., Negrón, J. F. \& Seybold. S. J. (2010). Climate Change and Bark Beetles of the Western United States and Canada: Direct and Indirect Effects. Bio-Science 60:602-613. DOI: $10.1525 /$ bio. 2010.60 .8 .6

Berrueta Soriano, V. M. (2007). Evaluación energética del desempeño de dispositivos para la cocción con leña. UNAM, México, D.F.

Billings, R. F., Clarke, R. S., Espino Mendoza, V., Cordón-Cabrera, 
P., Meléndez Figueroa, J. R., Campos, J. R. \& Baeza, G. (2004). Bark beetle outbreaks and fire: a devastating combination for Central America's pine forests. Unasylva 55:15-21.

Bray, D. B., Merino, P. L. \& Barry, D. (2007). Los bosques comunitarios de México: manejo sustentable de paisajes forestales. Instituto Nacional de Ecología, México, D.F.

Brolsma, R. J. (2010). Effect of climate change on temperate forest ecosystems. Royal Dutch Geographical Society / Faculty of Geosciences, Utrecht University, Utrecht.

Challenger, A. \& Soberón, J. (2008). Los ecosistemas terrestres. Pages 87-108 in Conabio, editor. Capital natural de México, vol. 1: Conocimiento actual de la biodiversidad. CONABIO.

Christiansen, E. \& Bakke,A. (1988). The spruce bark beetle of Eurasia. Pages 479-503 in A. A. Berryman, editor. Dynamics of Forest Insect Populations: Patterns, Causes, Implications. Plenum, New York.

Cibrián-Tovar, D., Méndez, J. T., Campos, R., Yates III, O. \& Flores, J. (1995). Insectos Forestales de México/Forest Insects of México. UniversidadAutónoma Chapingo. SARH.USDA.Natural Resources Canada. Comisión Forestal deAmérica del Norte. FAO.

CONABIO. (2009). Capital Natural de México. Comisión Nacional para el Conocimiento y Uso de la Biodiversidad.

Cudmore, T. J., Björklund, N., Carroll, A. L. \& Staffan Lindgren, B. (2010). Climate change and range expansion of an aggressive bark beetle: evidence of higher beetle reproduction in natve host tree populations. Journal of Applied Ecology 47:1036-1043. DOI: 10.1111/j.1365-2664.2010.01848.x

Dhar,A., Parrott, L. \& Hawkins, C. D. B. (2016(.Aftermath of Mountain Pine Beetle Outbreak in British Columbia: Stand Dynamics, Management Response and Ecosystem Resilience. Forests 7:119. DOI: $10.3390 / \mathrm{f} 7080171$

Etaough Jones, M., Paine, T. D., Fenn, M. E. \& Poth, M. A. (2004). Influence of ozone and nitrogen deposition on bark beetle activity under drought conditions. Forest Ecology and Management 200:67-76. DOI: 10.1016/j.foreco.2004.06.003

Faccoli, M. (2002). Winter mortality in sub-corticolous populations of Ips typographus (Coleoptera, Scolytidae) and tis parasitoird in the south-eastern Alps. Journal of Pest Science 75:62-68. DOI: 10.1034/j.1399-5448.2002.02017.x

Farrel, B. D., Squeira, A. S., O'Meara, B. C., Normark, B. B., Chung, J. H. \& Jordal, B. H. (2001). The evolution of agriculture in beetles (Curculionidae: Scolytinae adn Platypodinae). Evolution 55:20112027. DOI: 10.1111/j.0014-3820.2001.tb01318.x

Fonseca, G. J., de los Santos-Posadas, H., Llanderal, C. C., CibriánTovar, D., Rodríguez, T. D. \& Vargas, H. J. (2008). Ips e insectos barrenadores en árboles de Pinus montezumae dañados por incendios. Madera y bosques 14:69-80.

Gaylord, M. L., Kolb, T. E. \& McDowell, N. (2015). Mechanisms of piñon pine mortality after severe drought: a retrospective study of mature trees. Tree Physiology 35:806-816. DOI: 10.1093/ treephys/tpv038

González, P., Neilson, R. P., Lenihan, J. M. \& Drapek, R. J. (2010). Global patterns in the vulnerability of ecossytems to vegetation shifts due to climate change. Global Ecology and Biogeography:1-14. DOI: 10.1111/j.1466-8238.2010.00558.x

Grace, J., Beringer, F. \& Nagy, L. (2002). Impacts of climate change on the tree line. Annals of Botany 90:537-544. DOI: 10.1093/ aob/mcf 222

Kurz, W. A., Dymond, C. C., Stinson, G., Rampley, G. J., Neilson, E. T., Carroll, A. L., Ebata, T. \& Safranyik, L. (2003). Mountain pine beetle and forest carbon feedback to climate change. Nature 452:987-990. DOI: $10.1038 /$ nature 06777

Lenoir, J., Gégout, J. C., Marquet, P., de Ruffray, P. \& Brisse, H. (2008). A significant upward shift in plant species optimum elevation during the 20th Century. Science 320. DOI: 10.1126/ science. 1156831

Logan, J. A., Régnière, J. \& Powell, J. A. (2003). Assessing the impacts of global warming on forest pest dynamics. Frontiers in Ecology and the Environment 1:130-137. DOI: 10.1890/15409295 (2003)001[0130:ATIOGW]2.0.CO;2

Manzo-Delgado, L., López-García, J. \& Alcántara-Ayala, I. (2013). Role of forest conservation in lessening land degradation in a temperate region: The Monarch Butterfly Biosphere Reserve, Mexico. Journal of Environmental Management 138:55-66. DOI: 10.1016/j.jenvman.2013.11.017

Mátyás, C. (2010). Forecasts needed for retreating forests. Nature 469:1271. DOI: $10.1038 / 4641271 \mathrm{a}$

Návar, J. (2015). Hydro-climatic variability and perturbations in Mexico's north-western temperate forests. Ecohydrology 8:10651072. DOI: $10.1002 /$ eco. 1564

Negrón, J. F., McMillin, J. D., Anhold, J. A. \& Coulson, D. (2009). Bark beetle-caused mortality in a drought-affected ponderosa pine landscape in Arizona, USA. Forest Ecology and Management 257:1353-1362. DOI: 10.1016/j.foreco.2008.12.002

Negrón, J. F. \& Popp, J. B. (2004). Probability of ponderosa pine infestationby mountains pine beetle in the Colorado Front Range. Forest Ecology and Management 191:17-27. DOI: 10.1016/j. foreco.2003.10.026

Parmesan, C., Ryholm, N., Stefanescu, C., Holl, J. K., Thomas, C. D., Descimon, H., Huntley, B., Kaila, L., Kullberg, J., Tammaru, T., Tennent, W. J.,Thomas, J. A. \& Warren, M. (1999). Poleward shifts in geographical ranges of butterfly species associated with regional warming. Nature 399:579-583. DOI: 10.1038/21181

Parmesan, C. \& Yohe, G. (2003). A globally coherent fingerprint of climate change impacts across natural systems. Nature 421:3742. DOI:

Pausas, J. G. \& Keeley, J. E. (2009). A Burning Story: The Role of Fire in the History of Life. Bio-Science 59:593-601. DOI: 10.1525/ bio.2009.59.7.10

Pavia, E. G., Graef, F. \& Reyes, J. (2009). Annual and seasonal surface air temperature trends in Mexico. International Journal of Climatology 29:1324-1329. DOI: 10.1002/joc.1787

Pérez-Salicrup, D. R., Cantú-Fernández, M., Jaramillo-López, P. F., Carlón-Allende, T., Sáenz-Ceja, E., Garduño-Mendoza, E. \& Martínez-Torres, L. (2016). Restauración de un proceso: el fuego en la Reserva de la Biosfera Mariposa Monarca en los estados de México y Michoacán. Experiencias mexicanas en la restauración de los ecosistemas. UNAM, CRIM-UEAM, CONABIO, Cuernavaca, Morelos.

Raffa, K. F., Aukema, B. H., Bentz, B. J., Carroll, A. L., Hicke, J. A., Turner, M. G. \& Romme, W. H. (2008). Cross-scale Drivers of Natural Disturbances Prone to Anthropogenic Amplification: The Dynamics of Bark Beetle Eruptions. Bio-Science 58:501-517. DOI: 10.1641/B580607

Reeve, J. D. (1997). Predation and bark beetle dynamics. Oecologia 112:48-54. DOI: 10.1007/s004420050282

Rehfeldt, G. E., Crookston, N. L., Sáenz-Romero, C. \& Campbel, E. (2012). North American vegetation model for land use planning in a changing climate: A statistical solution to large classification 
problems. Ecological Applications 22:119-141. DOI: 10.1007/ s10584-009-9753-5

Reich, R. M., Lundquist, J. E. \& Hughes, K. (2016). Host-environment mismatches associated with subalpine fir decline in Colorado. Journal of Forestry Research 27:1177-1189. DOI: 10.1007/ s11676-016-0234-1

Rodríguez-Trejo, D. A. (2008). Fire regimes, fire ecology, and fire management in Mexico. Ambio 37:548-556. DOI: 10.1579/00447447-37.7.548

Rubín-Aguirre, A., Sáenz-Romero, C., Lindig-Cisneros, R., delRío-Mora, A. A., Tena-Morelos, C. A., Campos-Bolaños, R. \& del-Val, E. (2015). Bark beetle pests in an altitudinal gradient of a Mexican managed forest. Forest Ecology and Management 343:73-79. DOI: 10.1016/j.foreco.2015.01.028

Ryall, K. L. \& Fahrig, L. (2005). Habitat loss decreases predator-prey ratios in a pine-bark beetle system. Oikos 110:265-270. DOI: 10.1111/j.0030-1299.2005.13691.x

Rzedowski, J. (1978). Vegetación de México. Limusa, México, D.F.

Sáenz-Romero, C., Rehfeldt, G. E., Crookston, N. L., Duval, P. \& Beaulieu, J. (2010). Spline models of contemporary, 2030, 2060 and 2090 climates for Mexico and their use in understanding climate-change impacts on the vegetation. Climatic Change 102:595-623. DOI: 10.1007/s10584-009-9753-5

Safranyik, L., Carroll, A. L., Regniere, J., Langor, D. W., Riel, W. G., Shore, T. L., Peter, B., Cooke, B. J., Nealis, V. G. \& Taylor, S. W. (2010). Potential for range expansion of mountain pine beetle into the boreal forest of North America. Canadian Entomologist 142:415-442. DOI: 10.4039/n08-CPA01

Safranyik, L. \& Linton,D. A. (1998). Mortality of mountain pine beetle larvae, Dendroctonus ponderosae (Coleoptera: Scolytidae) in logs of lodgepole pine (Pinus contorta var. latifolia) at constant low temperatures. Journal of the Entomological Society of British Columbia 95:81-87.

Salinas-Moreno, Y., Ager, A., Vargas, C. F., Hayes, J. L. \& Zúñiga, G. (2010). Determining the vulnerability of Mexican pine forests to bark beetles of the genus Dendroctonus Erichson (Coleoptera: Curculionidae: Scolytinae). Forest Ecology and Management 260:52-61. DOI: 10.1016/j.foreco.2010.03.029

Salinas-Moreno, Y., Mendoza, M. G., Barrios, M. A., Cisneros, R., Macías-Sámano, J. \& Zúñiga, G. (2004). Areography of the genus Dendroctonus (Coleoptera: Curculionidae: Scolytinae) in Mexico. Journal of Biogeography 31:1163-1177. DOI: 10.1111/j.1365-2699.2004.01110.x

Sánchez-González, A. (2008). Una visión actual de la diversidad y distribución de los pinos de México. Madera y bosques 14:107-120.

Schutt, P. \& Cowling, E. B. (1985). Waldsterben, a general decline of forests in central Europe: symptoms, development, and possible causes. Plant Disease 69:548-558.

SEMARNAT. (2015). Anuario estadístico de la producción forestal 2014. SEMARNAT, México, D.F.

Smith, T. M., Leemans, R. \& Shugart, H. H. (1992). Sensitivity of terrestrial carbon storage to $\mathrm{CO}_{2}$-induced climate change comparison of 4 scenarios based on general-circulation models. Climatic Change 21:367-384. DOI: 10.1007/BF00141377

Ungerer, M. J., Ayres, M. P. \& Lombardero, M. J. (1999). Climate and the northern distribution limits of Dendroctonus frontalis Zimmerman (Coleoptera: Scolytidae). Journal of Biogeography 26:1133-1145. DOI: 10.1046/j.1365-2699.1999.00363.x

Walther, G. R., Post, E., Convey, P., Menzel, A., Parmesan, C., Beebee, T. J. C., Fromentin, J. M., Hoegh-Guldberg, O. \& Barilein, F. (2002). Ecological responses to recent climate change. Nature 416:389-395. DOI: $10.1038 / 416389^{\mathrm{a}}$

Wilson, R. J., Gutiérrez, D., Gutiérrez, J., Martínez, D., Agudo, R. \& Monserrat, V. J. (2005). Changes to the elevational limits and extent of species ranges associated with climate change. Ecology Letters 8:1138-1146. DOI: 10.1111/j.1461-0248.2005.00824.x

Winter, M. B., Baier, R. \& Ammer, C. (2015). Regeneration dynamics and resilience of unmanaged mountain forests in the Northern Limestone Alps following bark beetle-induced spruce dieback. European Journal of Forest Research 134:949-968. DOI: 10.1007/ s10342-015-0901-3

Wood, S. L. (1982). The bark and ambrosia beetles of North and Central America(Coleoptera: Scolytidae), a taxonomic monograph. Great Basin Natural Memories 6:1-1359.

World Metereological Organization. (2013). The global climate 20012010; a decade of climate extremes; summary report. World Metereological Organization, Génova, Suiza.

Worral, J. J., Rehfeldt, G. E., Hamann, A., Hogg, E. H., Marchetti, S. B., Michaelian, M. \& Gray, L. K. (2013). Recent declines of Populus tremuloides in North America linked to climate. Forest Ecology and Management 299:35-51. DOI: 10.1016/j.foreco.2012.12.033 